\title{
Active Sportswear Fabrics
}

\author{
Vishal Kejkar ${ }^{1 *}$ and Rajesh Dhore ${ }^{2}$ \\ ${ }^{1} \mathrm{M}$ Tech student in SVITT, India \\ ${ }^{2}$ Assistance Professor, India
}

ISSN: 2578-0271

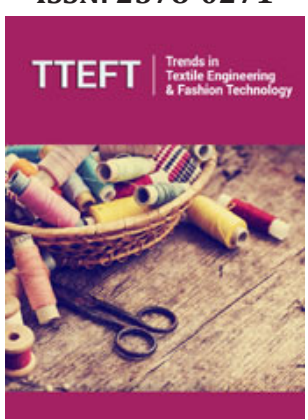

*Corresponding author: Vishal Kejkar, M Tech student in SVITT, India

Submission: 侮 May 05, 2019

Published: 湢 May 29, 2019

Volume 5 - Issue 2

How to cite this article: Vishal $\mathrm{K}$, Rajesh D. Active Sportswear Fabrics. Advancements Bioequiv Availab.5(2) TTEFT.000606.2019.

DOI: 10.31031/TTEFT.2019.05.000606

Copyright@ Vishal Kejkar, This article is distributed under the terms of the Creative Commons Attribution 4.0 International License, which permits unrestricted use and redistribution provided that the original author and source are credited.

\begin{abstract}
In recent years, innovation in active sportswear has been progressing to perform high functions and to gain comfort. Therefore, sportswear industries keep their attention on market diversification for fibrous materials and on textile science and technology. The use of innovative textile science and technology in the manufacturing of sports and leisurewear fabrics is continuously improving day by day to enhance the requirements for sports activities for their better performance in the sports. The factors consider for developing active sportswear fabrics are polymer and fiber technology, fabric and yarn production techniques, and finishing techniques to obtain high performance fiber, modified structure of yarns and fabrics. The present paper is concerned with the review of fiber types and their properties, fabric types and properties and works done in development of comfort properties of sportswear.
\end{abstract}

Keywords: Active sportswear fabrics; leisurewear fabrics; Sportswear; Sport tech; Technical textiles

\section{Introduction}

The people are showing their keen interest in sports activity and that the demand for sportswear continues to expand day by day, and those engaged in developing sportswear solutions in this field must therefore feel encouraged to produce an adequate response to these increasingly demanding expectations [1]. The consumers spend considerable amounts of money on functional sportswear fabrics, which have made the market more demanding to many producers. In regards this many researchers and industries are work together to enhance the properties of sports fabrics and make it active sportswear [2]. The properties require in sports fabrics are light weight, fast drying and elasticity properties [1]. For making active sportswear it requires some functional, aesthetic and comfort properties like optimum heat and moisture management properties, good air and water permeability, prevention of a long term feeling of dampness, low water absorption of the layer of clothing facing the skin, quick drying fabric to prevent catching cold, pleasant to skin, soft, non-abrasive and nonchafing, dimensionally stable even when wet, durable, lightweight, soft and pleasant touch, easy care, smart and functional design. Also has superior strength and durability [2]. Different sports demand the widely different performance in sportswear. Functional performance of sportswear depends on the type of sport, environmental conditions and amount of physical work required [3]. Wear performance of high active sportswear may affect the comfort level of player and hence it becomes one of the most important quality criterions [4]. The objective of this paper is to review the past research work carried out in the area of wear comfort and performance enhancement by high active sportswear.

\section{Need of active sports wear}

In sports, previously a conventional apparel textile technology was used. For that the player must face so many problems [1] such as:

a) Sweating - which stick the fabric with body

b) Feeling hot during run

c) Improper stretch ability

d) Extra weight of the fabric after moisture absorption

e) Give protection to body skin during fall on the ground 
f) Inadequate fabric for fluid resistance for swimmer

g) Windproof, waterproof fabric for sailor

h) Light weight shoe with proper strength \& air circulation

i) High tenacity \& resistance to abrasion for the skiwear uniform

j) Improper gripping of the shoes during run etc.

Therefore, currently technology must develop in sportswear to overcome on the above cited limitations in sportswear fabric. In regards this, many researchers' works on improving the comfort performance of active sportswear. The countries that were in the foremost are: Japan, Sweden, Spain and United State and some research centers as well as the manufacturer who are leading in the sports textiles are: Kanebo Ltd., Unitika Ltd., DuPont, W. L.GORE, Toyboo Co. Ltd., Teijin and Triangle research Development Corporation [4].

\section{Comfort characteristics required for sportswear}

There are four types of comfort characteristics require in active sportswear for functional performance are - thermo-physiological comfort, skin sensorial comfort, ergonomics wear comfort and psychological comfort.

Thermo physiological comfort: This type of comfort is related to the thermal balance of body i.e. when the rate of loss of heat from the body equals to the rate of generation of heat by the various physiological processes. It determines the breathability and moisture management properties of fabric i.e. heat and moisture transport through the fabric.

Sensorial/Tactile comfort: This type of the comfort is related to the way that the person feels when apparel is worn next to skin rather than temperature balance. This comfort is mainly depending on the fabric surface, fabric structure and to some extent, moisture transport capacity. It is linked with skin contact sensations and is often expressed as feelings of smoothness and softness.

Mobility/Dexterity: The considerable movements of body parts occur, while doing the various physical activities in various active sports. Therefore, active sportswear must have elasticity properties (mobility) to move freely.

Psychological comfort: Psychological comfort is related to the feeling of the wearer. Psychological comfort is determined by the aesthetic properties from the point of view of style or fashion.

Human body tries to maintain the inner body core temperature at $37 \pm 1^{\circ} \mathrm{C}$. This core temperature is maintained by the body through four ways of heat transfer, viz conduction, convection, radiation and evaporation. During sports activity, $80 \%$ of energy is converted into heat and in case of hot conditions when air temperature is higher than body core temperature, heat loss is carried out through evaporation for maintaining the body temperature. The heat loss amount depends on the rate of sweat evaporation in environment. But the evaporation rate is determined by the atmospheric conditions [5]. In warmer areas, sweat rate can go up to as high as $2.5 \mathrm{~L} / \mathrm{h}$ due to high metabolic activities in active sportswear, which results into increasing convective and radiative heat loads. The researchers found that fabric with higher moisture absorption increases the performance of players [6]. Fabrics provide a microclimate between the body and the atmosphere and acts as a courier for heat and vapour transfer between the skin and the environment. The moisture transmission through the fabric is carried out by mainly three processes like diffusion of moisture, sorption's-desorption and forced convection by moving air close to skin. In hot and humid conditions, clothing should rapidly absorb the moisture and instantaneously transport it through the fabric in environment through wetting and wicking actions so that the player feels comfortable. The initial action is called as wetting action which involved the spreading of fluid on textiles and fibreliquid spaces replaces the fibre-air interface in fabric. The wicking action is carried out by fibre-liquid molecular attraction at the fabric surface and determined by the surface tension and the effective capillary pathways and pore distribution on fibres. The physical comfort of a body is related to the skin temperature and moisture content in environment. Skin temperature and moisture content of microclimate are strongly stimulated by the level of physical effort. The fabric type has a significant effect on moisture of skin. A study showed that moisture vapour resistance and moisture accumulation within clothing determines the comfort of sportswear during sports. Clothing thermal insulation decreases during perspiration, and the amount of reduction varies from $2 \%$ to $8 \%$, due to accumulation of water within clothing, which can cause the "after chill" effect to wearers after heavy exercise. Skin sensorial or tactile comfort of sportswear is determined by surface friction, roughness and softness. Skin is the largest organ of human body and is more prone to injury in sports. Chafing is the most common problem in active sports which occurs due to mechanical rubbing of the skin with clothing or other parts of skin. Skin abrasion is another skin injury which removal of skin cells during is rubbing with external surfaces like synthetic turf. Other skin problem like Urticarial and hives are also reported due to repeated rubbing between skin and ill-fitting apparel. It has been reported that these types of injuries can be reduced by selecting well-fitted and low friction sportswear. Ergonomic comfort determines the fit and freedom of movement which depends upon the fit design, fabric elasticity and pattern construction. In active sports like running, skin extension and contraction take place due to high degree of body movement which alters the corresponding body measurements. Sportswear clothing should not restrain these movements else discomfort will be created due to undesired garment pressure on body. Elastic fabric is commonly used in tight-fit running shorts which provide the desired shape and size with adequate room for body movements.

\section{Functional requirements of high active sportswear}

In high active sports, heat stress is of great concern due to high level of metabolic heat generation which is in the range of $800-1300 \mathrm{~W}$. This amount of heat can increase the body core temperature by $1.5-2^{\circ} \mathrm{C}$. To control the core temperature of body, sweat generation takes place and heat of vaporization of water is used to give the cooling effect4. Sweat generation can go as high 
as $2.5 \mathrm{~L} / \mathrm{h}$ and hence the main functional requirement of high active sportswear is sweat absorbing, fast drying and cooling. High active sportswear should also have high stretch and elastic recovery to provide enough fit and freedom of movement to the wearer. In number of active sports like jumping, running and power lifting, compression is created by stretchable fabric to enhance the performance of an athlete. Compression Athletic Wear (CAW) provides the necessary compression and anatomic fit to an athlete. They are also known as "skin suits" as they conform to the natural curves of the body acting as second skin. Other requirements laid down for active sportswear are smoothness, softness, UV resistance, light weight, and easy care.

\section{Textile material and fabric structure for sportswear}

It is not possible to achieve all required properties for sportswear in a simple structure of any single fiber. The right type of fiber should be in the right place. The behavior of the fabric is mainly depending on its base fiber's properties. The most important properties are fiber type; weave construction; weight or thickness of the material and presence of chemical treatments [7-9]. Fibers for Active Sports Wear

Polyester: Polyester has outstanding dimensional stability and offer excellent resistance to dirt, Alkalis, decay, Mold and most common organic solvents. Excellent heat resistance or thermal stability is also an attribute of polyester. It is the fibre used It is the fibre used most commonly in base fabrics for active wear because of its low moisture absorption, easy care properties and low cost [2].

Polypropylene: Polypropylene is claimed to be a proved performer in moisture management due to its hydrophobic nature and has very good thermal characteristics, keeping the wearer warm in cold weather and cold in warm weather.

Nylon: Nylon fibre characteristic include lightweight, high strength and softness with good durability and has better wicking behavior. It is most often used in tightly woven outerwear, which can trap heat because of low air permeability.

Cotton: Cotton garments provide a good combination of softness and comfort. However, cotton is not recommended for use in base layer clothing because of its tendency to absorb and retain moisture [2].

Vicose rayon: The viscose rayon is not preferred next to skin as it holds water (13\% moisture regains) in sportswear. The outer layer of knitted hydrophilic portion of the twin layer Sportswear can be of viscose rayon, which absorbs 2-3 times more moisture than cotton.

Hygra 20: Unitika Limited has launched Hygra, which is a sheath core type filament yarn composed of fibre made from water absorbing polymer and nylon. The water-absorbing polymer has a special network structure that absorbs 35 times its own weight of water and offers quick releasing properties that the conventional water absorbing polymer cannot do. Hygra also has superior antistatic properties even under low wet conditions. The main apparel applications include sportswear like athletic wear, skiwear, golf wear etc.

Killat N23: Killat $\mathrm{N}$ from Kanebo Ltd. is a nylon hollow filament. The hollow portion is about 33 per cent of the cross section of each filament due to which it gives good water absorbency and warmth retentive property. The manufacturing technology of Killat $\mathrm{N}$ is very interesting. The yarn is spun as bi-component filament yarn with soluble polyester copolymer as the core portion and nylon as the skin portion.

Lycra25: Lycra, a truly synthetic fibre of long chain polymer composed of at least $85 \%$ segmented polyurethane, finds wide range of end users such as swimwear, active sportswear, floor gymnastics because of its comfort and fit20. Adding Lycra to a fabric gives it stretch and recovery, particularly in gymnastics and swimwear where body skin flexing and stretching are inevitable. Lycra T-9026 requires still effort for the same extensibility [8].

Dacron: 4-Channel Polyester a generic term for a high performance four channel fibre engineered to move moisture and speed the evaporation of perspiration. It is a superior fabric for wicking action, drying time, moisture absorption and transport.

Tencel: Tencel is the generic name of Lyocell. Lyocell is a natural, manmade fibre produced in an environment friendly process from wood pulp that has become popular in clothing. The moisture management of Tencel is unique when compared to synthetic fibres and allows for peak performances in sports. The excellent moisture absorption is perfect for the skin and thus guarantees wellbeing at a very high level.

Bamboo: Bamboo fabrics are made from pure bamboo fibre yarns which have excellent wet permeability, moisture vapour transmission property, soft hand, better drape, easy dyeing, and splendid colors. It is newly founded, great prospective green fabric. Bamboo fibre has a unique function of anti-bacteria, which is suitable to make underwear, tight t- shirt and socks. Its anti ultraviolet nature is suitable to make summer clothing.

Soybean: Soybean protein contained in the fibre remakes a superior, soft hand endowed with both moisture absorbency and permeability, which makes best application in knits and innerwear. Finishes with an antibacterial agent, health-care functionalities are also given. It has great potential in its use in high-grade knits and innerwear.

\section{Development in polymer}

"Phase Change" materials (PCM)" are being developed for sportswear. This means that they contain a chemical that changes from being a liquid to a gel at around body temperature. This alters the fabric's insulation properties so that you can design clothes that keep the body at a constant temperature no matter what happens to the air temperature. These are used in cold weather protective clothing like gloves, boots and hats. Phase change stuff produce microcapsules full of paraffin. When the capsules are heated, the paraffin liquidities and heat energy is stored. When the environment cools, the paraffin crystallizes again, releasing heat. The paraffin, which is available in different recipes with various melting points, is referred to the phase change material or PCM. Because the paraffin 
can be formulated with different melting points, the microcapsules can be designed to keep the wearer either warm or cool [10-12].

\section{Types of fabrics used in sportswear}

A wide range of woven, knitted and nonwoven fabrics are commercially available for sportswear. These fabrics differ in their structure such as entrapped air, pore shape and size, bulk and surface properties etc. which may affect the heat and moisture transmission characteristics of the fabrics [3]. Generally, for sportswear, knitted fabrics are preferred as these fabrics have greater elasticity and stretch ability compared to woven fabrics, which provide unrestricted freedom of movement and transmission of body vapor to the next textile layer in the clothing system. With new combinations of fabrics and yarns, and with developments in fabric construction, knitted fabric appears to be the ideal base for active sportswear [13]. Knitted garments are mainly worn next to the skin and therefore deserve attention. But the major problem in both woven and knitted fabrics is that, owing to sweat generation, the fabric tends to stick with the body of the athlete resulting in discomfort during his running. The term "sportswear" refers to any garment specially designed for use in sport activities. In order to maximize the athlete's performance, the sportswear must be efficient, essentially, in body thermoregulation and moisture management. All these factors are dependent on the fibrous materials and structures used [5].

\section{Modification in fabric structure}

Waterproof breathable fabrics: In the last few years, the requirements of fabrics are lightweight, but durable; waterproof, but breathable and soft but more resistant. In this way, "smart fabrics" appeared to answer to some of these requirements [5]. These fabrics were designed and developed to maximize the benefits of physical activity and minimize their possible drawbacks. Fibrous structures used in the production of more casual wear are of elastic type, in order to ensure an unlimited freedom of movement and carry moisture from the body to the next fibrous layers. With the right combination of fibers, yarns and technology, it is possible to produce ideal structures for functional sportswear [11].

Layered Fabrics: Layered fabrics became common for active sportswear. The performance of layered fabric in thermo physiological regulation is better than single layer textile structure. Each layer has distinct function; the layer next to the skin is to wick away the perspiration rapidly to the outer layer, which absorbs and dissipates it rapidly to the atmosphere by evaporation. In doing so, it takes away some of the body heat and keeps the body cool. On the inside, a synthetic material with good moisture transfer properties, e.g. polyester, nylon, acrylic or polypropylene is used whereas on the outside, a material which is good absorber of moisture, e.g. cotton, wool, viscose rayon or their blends can be placed [17]. The conductive inner layer transfers the liquid perspiration rapidly to the absorbent outer layer chiefly as a result of the capillary effect. The absorbent outside of the textile material functions as a buffer zone for excess perspiration. In another way of constructing a physiologically functional fabric is three-layer structure. The use of superfine or microfiber yarn enables production of dense fabrics leading to more effective capillary action, with better thermophysiological regulation $[13,14]$.

\section{Finishing treatment}

Functional sportswear was developed by graft polymerization process in which hydrophobic inner side and hydrophilic outer side of fabric was created by polymerization of acrylic acid on polyester fiber. High performance of moisture and odor control with effective dispersion of sweat was reported for this fabric. In another attempt, polyester fabric with incorporated activated carbon has been developed which shows improved moisture comfort due to absorption of sweat impulses by carbon particles. This type of sportswear is found to be more comfortable in mild physical activity but not in high strenuous exercise due to slower drying. Moisture management finishes enhance the comfort level in sportswear by rapid wicking and evaporation. MMF Resil HJHP increases the absorbency in polyester when used alone and gives better wicking when used with other finishes like Resil Nanocelle G6. The Nano dry finish for Nanotex LLC is also applied to sportswear to improve absorbency of sweat. The presence of either a microporous membrane or a laminating substrate in the double fabric assembly increases the vapour pressure build-up and a prolonged inner fabric surface temperature. Thicker, less porous membrane with smaller pore size increases rate of moisture vapour build-up in microclimate and gives longest peak time of surface temperature. Sportswear gets easily contaminated by perspiration which leads to bacterial growth. Fragrance finishes like microencapsulated peppermint is claimed to have muscle easing properties and antimicrobial finishes like beta cyclodextrin kills bacteria.

\section{Performance enhancement by sportswear}

Heat stress can be reduced by suitable choice of sportswear. Differences in heat stress were reported with different types of sportswear by measuring mean skin temperature change, mean core temperature change, heart rate and total sweat loss [15]. Moisture absorption of sportswear can influence the physiological responses and exercise performance. It was reported that clothing with higher moisture absorption enhances the performance and pitching speed of baseball players. A good correlation between moisture transmission of fabric and athlete's physiological response has been reported. A fabric with better moisture management properties increases athlete's cardio-respiratory fitness and performance [14]. Color of sportswear can also influence the performance of player. It has been reported that red color enhances the performance by stimuli of the testosterone dependent signal. The Compression Athletic Wear (CAW) improves the performance of players in number of active sports like jumping, power lifting and running. An athlete showed faster running speed over $10 \mathrm{~km}$ with lower heart rates and reduced post exercise muscle soreness when wearing CAW as compared to control. Compression garments are reported to cause reduction in metabolic energy cost of running at specific speed and improved sub maximal running economy. It has been reported that compression shorts resist fatigue due to reduced muscle oscillation during landing in sports like sprints 
or jump events. Enhanced performance by using CAW may be due to improved venous return and cardiac input which would reduce cardiovascular stress on athlete. Another mechanism of improved performance has been proposed which suggested that athletes run at a faster speed due to increased lactate clearance and improved leg power using CAW. Biomimetic swimwear is reported to increase the swimmer's speed with reduced net active drag force as compared to normal swimsuit [16,12].

\section{Innovative sportswear products}

Some high performance fibres like Coolmax®, Thermolite and Thermocool are being offered by Advanced Fibre Technology (ADVANSA) for various sportswear applications. Coolmax $\AA$ active is a high tech fibre made from specially engineered four channeled and six channeled polyester fiber which forms a transport system with an increased surface area that pulls moisture away from the skin to the outer layer of the fabric and keeps the wearer cool and dry. For active sports in cold climate, Thermolite ${ }^{\circledR}$ is very popular. Hollow core fibres trap in air for great insulation and provide warmth and comfort without weight [10]. The large surface area allows the fast evaporation of perspiration and wearer stays dry $50 \%$ faster than cotton. Channeled surface has been combined with hollow core in Coolmax® all season which move moisture away from the skin when wearer is hot as well as provide warmth for added comfort in colder days. Sport wool $®$ is a unique fabric developed for active sportswear by CSIRO Australia. It is basically a two-layer moisture management fabric with wool on the inner side and synthetic fibre on the outer side. Another innovation of CSIRO is quick dry wool with water resistant finish on wool which dramatically reduces the drying time. Blends of wool and moisture management fibres such as Coolmax and Fine cool have been studied to produce innovative yarns with specific functionalities. It was found that fabrics with coolmax fibres show the best capillary performance with quick transport of the perspiration from skin to environment. Fine cool fabrics show higher drying rate with quick drying after wetting [5]. Wool-based fabrics show lower absorption rate but good drying capacity. Toray offered many moisture management fabrics like Stunner $Q D \AA$ and Field sensor $\AA$. Stunner $Q D \AA$ is nylon woven fabric which quickly absorbs, disperses and evaporates perspiration for quick drying. Field sensor TM® has brushed inner side which provides insulation and moisture management, thus making it suitable for winter active sportswear. Field sensor $\mathrm{R} \circledast$ is ecofriendly moisture management fabric made from recycled polyester fibres which quickly absorb perspiration, carry it rapidly to fabric outer surface and disperse throughout outer surface for rapid evaporation. Fabric coating with micro-encapsulated phase changing material has been exploited by outlast. Products with Outlast technology buffer changes in humidity and temperature in microclimate and external environment. They maintain constant body temperature by absorbing excess body heat when temperature starts rising due to heat production and releasing it when temperature falls during cooling. Inotek $®$ fibre is innovative biomimetic $[16,17]$. When it absorbs moisture, it shrinks to thin structure causing microscopic air pockets to open and increase the breathability. This response is reversible and fibres come back to original dimension in dry conditions. Skin ${ }^{\circledR} 400$ series is elastane incorporated warp knitted innovative compression athletic wear which can increase the oxygen delivery to active muscles by dynamic gradient compression. Biomimetic swimsuit Fast skin developed by Speedo ${ }^{\circledR}$ is inspired from shark skin. The denticles of shark's skin and super stretch property of fabric can enhance the performance of swimmer by shape retention, muscle compression and reduced drag coefficient.

\section{Conclusion}

High active sportswear is very vast and challenging field in which required functionality can be designed by suitable choice of raw material, structure and geometry of fibres, yarns and fabrics, surface modification and garment assembly technique. During designing the sportswear fabrics, the aspects which are considered: protection/safety functions to protect wearers from adverse weather, comfort functions which gives wear comfort (thermal, sensorial and body movement comfort), exercise function to enhance performance of athlete, and aesthetic appeal and high fashion ability. Moisture management properties like sweat absorption sweat dissipation and faster drying are primary desirable functions of high active sportswear which affect the comfort sensation of player during the game. The required functions of sportswear fabrics differ in different situations such as type of sports, environmental conditions and level of activity etc. Sportswear developed by using special type of polymer, type of fibrous material, modifying the fibre/yarn/fabric structure, lamination, finishing technology and manufacturing technology etc. The introduction of high functionality and comfort in the sportswear fabrics provide unlimited scope for sportswear fabrics.

\section{References}

1. Manshahia M, Das A (2014) High active sportswear-a critical review. Indian Journal of Fibre \& Textile Research 39(4): 441-449.

2. Yonenaga A (1998) Engineered fabric for active and comfort sportswear, itb int. Text Bulletin 4: 22-26.

3. Kanakaraj P (2015) Active knit fabrics-functional need of sportswear application. Journal of Textile and Apparel Technology and Management 9(2): 1-11.

4. Ishtiaque SM, Gupta S (2000) Proceedings on smart textiles, their production and market strategies. 9: 58-64.

5. Kothari VK (2003) Fibres and fabrics for active sportswear. Asian Textile Journal 12(3): 55-61.

6. Sule AD, Sarkar RK, Bardhan MK (2004) Development of sportswear for Indian conditions. Manmade Textiles in India, pp. 123-129.

7. Meinander H (1995) Heat stress in sportswear. Text 26(8): 108-110.

8. Umbach KH (1993) Aspects of clothing physiology in the development of sportswear. Knitting Technique 15(3): 165-169.

9. Silva AP, Anand SC (2000) Responsive garments for sportswear, proceedings on smart textiles their production and market strategies. NIFT, pp. 32-49.

10. Sule AD, Bardhan MK, Sarkar RK (2004) Development of sportswear for Indian conditions. Man Made Textile in India, pp. 123-129. 
11. Behera BK, Mani MP, Mondal AK, Sharma N (2002) Comfort behaviour of cotton polypropylene based bilayer knitted fabrics. Asian Text J, pp. 61-67.

12. Chaudhari SS, Chitnis RS, Ramkrishnan R (2005) Waterproof breathable active sportswear fabrics. The Synthetic and Art Silk Mills Research, Mumbai.

13. Rognoni U (2001) Active sportswear fabrics, high functions and comfort. JTN The Int Text Magazine 554: 34-76.

14. Umbach KH (1993) Aspects of clothing physiology in the development of sportswear. Knitting Technique 15(3): 165-169.
15. Sule AD, Bardhan MK, Sarkar RK (2004) Development of sportswear for Indian conditions. Man Made Text, pp. 123-129.

16. Anon (2001) Active sportswear fabrics. Japan Textile News 559: 34-35.

17. Hari Gopalakrishnan, Ramachandran T (2016) study on comfort characteristics of three-layered technical textiles for sportswear, international conference on systems, science. Control Communication Engineering and Technology, USA.

For possible submissions Click below: 